\title{
Chameau HAT and DRpd3 HDAC function as antagonistic cofactors of JNK/AP-1-dependent transcription during Drosophila metamorphosis
}

\author{
Benoit Miotto, ${ }^{1,3}$ Thierry Sagnier, ${ }^{1}$ Hélène Berenger, ${ }^{1}$ Dirk Bohmann, ${ }^{2}$ Jacques Pradel, ${ }^{1,4}$ and \\ Yacine Graba ${ }^{1,5}$ \\ ${ }^{1}$ Laboratoire de Génétique et Physiologie du Développement, Institut de Biologie du Développement de Marseille, \\ CNRS/INSERM/Université de la Méditerranée, Parc Scientifique de Luminy, 13288 Marseille Cedex 9, France; ${ }^{2}$ Department \\ of Biomedical Genetics, University of Rochester, Rochester, New York 14642, USA
}

\begin{abstract}
Gene regulation by AP-1 transcription factors in response to Jun N-terminal kinase (JNK) signaling controls essential cellular processes during development and in pathological situations. Here, we report genetic and molecular evidence that the histone acetyltransferase (HAT) Chameau and the histone deacetylase DRpd3 act as antagonistic cofactors of DJun and DFos to modulate JNK-dependent transcription during thorax metamorphosis and JNK-induced apoptosis in Drosophila. We demonstrate in cultured cells that DFos phosphorylation mediated by JNK signaling plays a central role in coordinating the dynamics of Chameau and DRpd3 recruitment and function at AP-1-responsive promoters. Activating the pathway stimulates the HAT function of Chameau, promoting histone $\mathrm{H} 4$ acetylation and target gene transcription. Conversely, in response to JNK signaling inactivation, DRpd3 is recruited and suppresses histone acetylation and transcription. This study establishes a direct link among JNK signaling, DFos phosphorylation, chromatin modification, and AP-1-dependent transcription and its importance in a developing organism.
\end{abstract}

[Keywords: JNK signaling; Fos; histone acetylation; epigenetic control of transcription; Drosophila; development]

Supplemental material is available at http://www.genesdev.org.

Received July 15, 2005; revised version accepted October 31, 2005.

The transcriptional response to signal transduction via the evolutionary conserved Jun N-terminal kinase (JNK) pathway controls essential cellular processes, including morphogenesis, differentiation, and apoptosis during development and in physiopathological situations (Eferl and Wagner 2003; Xia and Karin 2004). A large body of research supports the model that in response to extracellular stimulation, JNK activates the transcriptional effector AP-1 by phosphorylation, and thereby reprograms target gene expression (Noselli and Agnes 1999; Kockel et al. 2001; Weston and Davis 2002). AP-1 mainly consists of Fos and Jun family proteins that can form homodimers or heterodimers and bind DNA through conserved bZIP domains. Given the importance of chroma-

\footnotetext{
${ }^{3}$ Present address: Harvard Medical School, Department of Biological Chemistry and Molecular Pharmacology, Boston, MA 02115, USA.

Corresponding authors.

${ }^{4}$ E-MAIL pradel@ibdm.univ-mrs.fr; FAX 33-4-91-82-06-82.

${ }^{5}$ E-MAIL graba@ibdm.univ-mrs.fr; FAX 33-4-91-82-06-82.

Article and publication are at http://www.genesdev.org/cgi/doi/10.1101/ gad.359506.
}

tin dynamics in the control of gene expression, recent work has focused on AP-1 interaction partners capable of chromatin modification and remodeling, notably enzymes able to reversibly modify histone tails by acetylation. The nuclear receptor corepressor (NCoR)/histone deacetylase 3 (HDAC3) complex was thus found to inhibit the JNK pathway (Zhang et al. 2002). The checkpoint function of the NCoR complex is relieved by c-Jun phosphorylation, which directs its removal from the promoter (Ogawa et al. 2004) and promotes the recruitment of the TAF7 RNA polymerase II (RNA pol II) subunit (Munz et al. 2003). AP-1 recruits DRpd3/HDAC1 to reverse histone acetylation at the promoter of the attacin- $A$ gene and its activation by the NF- $\mathrm{B}$ transcription factor Relish associated with the histone acetyltransferase (HAT) Pcaf (Kim et al. 2005). Other reports on stimulation of Fos or Jun activities by CBP (Bannister and Kouzarides 1995; Benkoussa et al. 2002) suggest that the recruitment of HAT coactivator complexes at target promoters mediate nucleosome acetylation and stimulate transcription. These findings, gained from experiments performed in vitro and in cultured cells, indicate that 
chromatin dynamics plays a central role in the cellular response to JNK signaling. However, the epigenetic mechanisms that control the transcriptional response in intact organisms remain unclear.

JNK signaling regulates a number of different processes during Drosophila development, including pupal thorax closure and apoptotic cell death in imaginal discs. The role of JNK signaling is well established in these two morphogenetic events. In the former, it drives the migration of wing discs toward the midline and their fusion into a continuous epidermal structure; in the latter, it apoptotically eliminates cells exposed to inappropriate proximodistal patterning cues (for review, see Kockel et al. 2001). The present study provides genetic and molecular evidence that Chameau (Chm), a MYST domain HAT previously reported to act in epigenetic mechanisms of transcriptional control (Grienenberger et al. 2000) and to support histone acetylation at replication origins (Aggarwal and Calvi 2004), and the HDAC DRpd3 modulate the transactivation potential of AP-1 during thorax closure and JNK-induced apoptosis in an antagonistic manner. Furthermore, by deciphering the mode of action of Chm and DRpd3 in vitro and in cultured cells, we provide mechanistic insights into the regulation of AP-1 function by chromatin.

\section{Results}

\section{Chm enhances INK signaling during thorax closure}

Animals homozygous for the amorphous allele $\mathrm{chm}^{14}$ lack zygotic chm function and die during late pupal stages. Pharate adults display morphological aberrations, especially a cleft of variable depth and width along the thorax midline. To quantitatively assess the strength of this phenotype, we adopted a system of four phenotypic classes, ranging from the absence to a mild, pronounced, or severe cleft (Fig. 1A). chm is expressed in the two epithelia of wing discs, the columnar epithelium (data not shown), and the peripodial membrane (Fig. 1B), and by $8 \mathrm{~h}$ after puparium formation (APF), when the two contralateral discs meet at the dorsal midline, transcription proceeds in fusion regions only (Fig. 1C). Together, the mutant phenotype and the expression pattern of $\mathrm{chm}$ support a function in migration and/or fusion of wing discs during metamorphosis.

As flies defective in JNK signaling display thorax defects similarly to chm mutants, we asked whether Chm might play a role in JNK signaling during thorax closure. Thus, we tested genetic interactions between $\mathrm{chm}$ and genes encoding components of the JNK pathway (Fig. 1D). Reducing the gene dosage of positive effectors, such as DINKK/hemipterous (hep) and Dfos/kayak (kay), does not per se affect thorax closure but exacerbates the $\mathrm{chm}$ phenotype: Heterozygosity for hep raises the proportion of class II phenotypes from $14 \%$ to $39 \%$; reducing $D$ fos activity has a more pronounced effect, as class III rises from $6 \%$ to $97 \%$ in the case of the null allele $\mathrm{kay}^{1}$ and to $40 \%$ with the hypomorphic allele $\mathrm{kay}^{2}$. Conversely, heterozygosity for puckered (puc), a repressor of JNK activ-
A

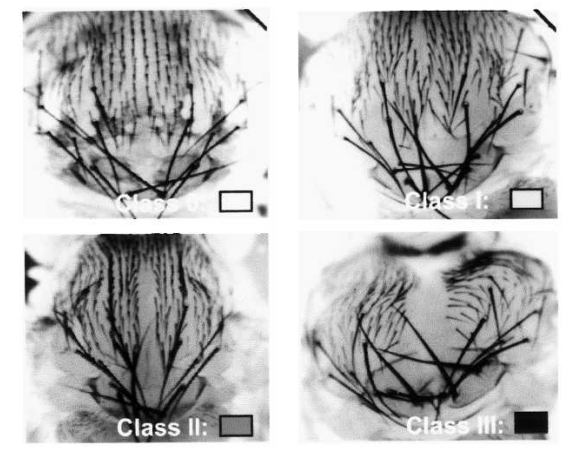

B

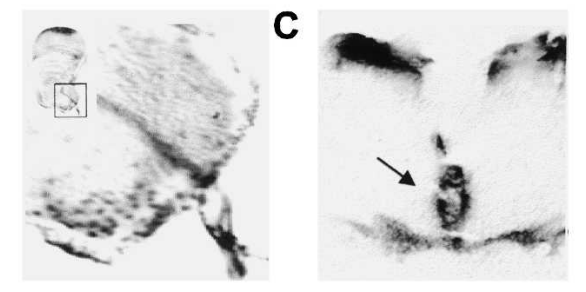

D

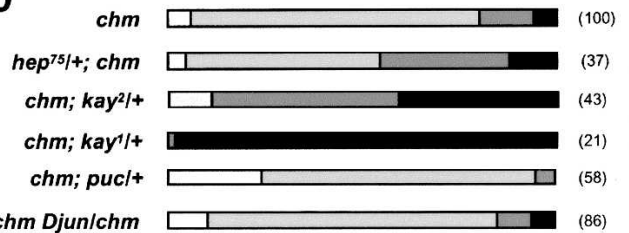

E
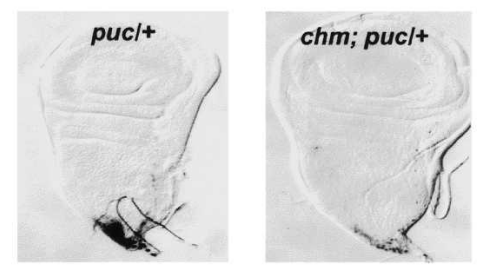

F

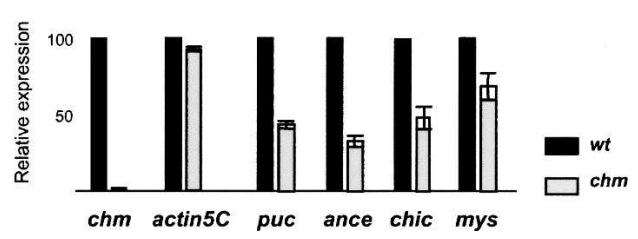

Figure 1. Chm enhances JNK signaling during thorax closure. (A) Variable penetrance of $\mathrm{chm}$ thoracic cleft phenotype. (B) $\mathrm{chm}$ expression in peripodial cells as revealed by $\beta$-Gal activity in the large peripodial nuclei of wing discs from the $\mathrm{chm}^{7 l a c z}$ enhancer trap line. The inset shows the region of the disc that has been magnified. $(C) \mathrm{chm}$ expression at sites of imaginal disc fusion revealed by in situ hybridization on dissected pupae $8 \mathrm{~h}$ APF. Arrow indicates the dorsal midline. $(D)$ Genetic interactions between $\mathrm{chm}$ and JNK pathway components encoding genes hep, kay, puc, and Diun, as revealed by changes in the strength of the cleft phenotype. For the color code representing the different phenotypic classes refer to $A$. The numbers of examined pupae are shown in parentheses. (E) puc expression in wild-type (left) and chm mutant (right) discs, revealed by $\beta$-Gal activity in the enhancer trap line $p u c^{E 69} .(F)$ Transcription levels of chm; of actin5C, which is not an AP-1 target; and of the target genes puc, mys, ance, and chic in wild-type and chm wing discs $1 \mathrm{~h} \mathrm{APF}$. 
ity, dominantly suppresses the thoracic cleft of $\mathrm{chm} \mathrm{mu-}$ tants, as no chm; puc/+ pharate adult presents a class III phenotype. Moreover, this genotype produces viable adult escapers, an effect already observed with lethal combinations of hep and Dfos alleles rescued by puc heterozygosity (Agnes et al. 1999; Zeitlinger and Bohmann 1999). These positive and negative genetic interactions indicate that $\mathrm{Chm}$ cooperates with JNK signaling during thorax closure. We noticed, however, that heterozygosity for Diun does not exacerbate the chm phenotype (Fig. 1D). The observation that Dfos, but not Diun, alleles dominantly interact with $\mathrm{chm}$ suggests that DFos acts independently of DJun in the context of Chm-dependent thorax closure processes.

Next, we examined AP-1 target gene transcription. The puc gene is activated by JNK signaling in proximal peripodial cells (Agnes et al. 1999; Zeitlinger and Bohmann 1999; Pastor-Pareja et al. 2004). In chm mutant discs, puc expression is strongly reduced but can still be detected, consistent with a stimulatory rather than essential function of $\mathrm{Chm}$ for JNK signaling in these cells (Fig. 1E). To confirm such a function, we compared transcription levels of puc and three additional bona fide JNK targets-ance, chickadee (chic), and myospheroid (mys) (Jasper et al. 2001)-in wild-type and chm mutant wing discs. Results from quantitative RT-PCR show that the transcription of the four target genes is significantly reduced in a chm background (Fig. 1F). We conclude that $\mathrm{Chm}$ supports JNK-dependent gene activation during wing disc development and thorax closure.

\section{Chm acts positively in the INK-dependent apoptotic pathway}

To determine whether chm contributes to JNK-dependent processes in addition to thorax closure, we examined JNK-induced apoptotic cell death. In the insertion trap line $h e p^{C A}$, JNK signaling is ectopically activated in the wing blade primordium, which causes cell death and induces notches of variable extent in the adult wing (Adachi-Yamada et al. 1999). Eliminating one copy of Diun, Dfos, or chm rescues the hep ${ }^{C A}$ wing phenotype. Furthermore, loss of one copy of chm partially reverts the enhancement of the hep ${ }^{C A}$ notching phenotype that is observed as a consequence of puc heterozygosity (Fig. 2A). Further supporting the notion of cooperation between JNK and Chm, cell death as detected by acridine orange staining in hep ${ }^{C A}$ wing discs is abrogated in chm homozygous conditions (Fig. 2B). JNK-dependent apoptosis is also induced in response to morphogen gradient distortion caused by misexpression of scalloped (sd) (Liu et al. 2000) or optomotor blind (omb) (Adachi-Yamada et al. 1999). We observed that heterozygosity for puc aggravates_-and for Diun, Dfos, or chm dominantly rescuesthe notched wing phenotype of flies heterozygous for $s d$ (Fig. 2C) or omb (data not shown). Thus, Chm acts as a positive effector in the JNK-regulated apoptotic pathway. Interestingly, in this process $\mathrm{chm}$ cooperates with both $D$ fos and Diun genes.
A
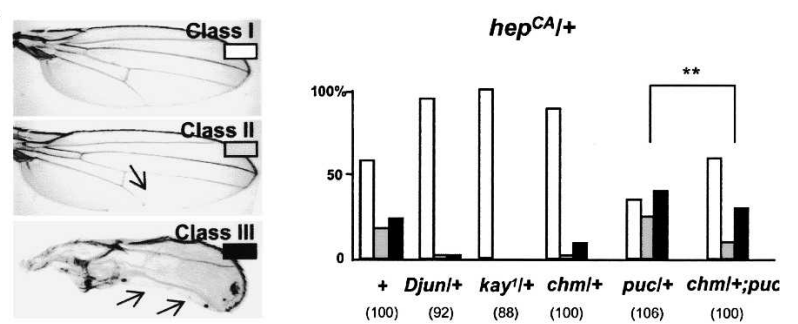

B

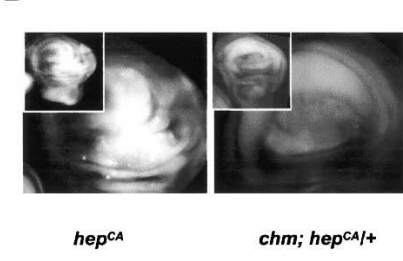

C $\quad s d^{\text {Gala/t+ }}$

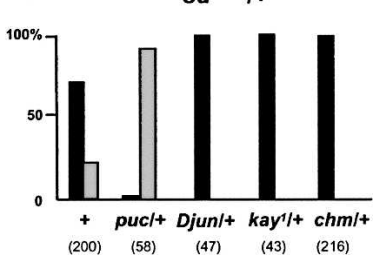

Figure 2. chm is required for JNK-induced apoptosis. (A) Variable severity of the notched wing phenotype induced by hep ${ }^{C A}$ (left, arrows show wing notches) and changes induced in the relative abundance of the phenotypic classes by heterozygosis for Diun, kay, puc, chm, and chm plus puc (right). (B) Acridine orange staining of hep ${ }^{C A}$ and $c h m$; hep $p^{C A} /+$ wing discs. $(C)$

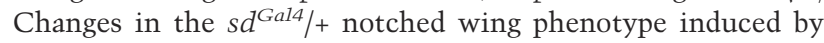
puc, Diun, kay, or chm heterozygosis. The numbers of examined wings are shown in parentheses.

\section{Chm acts as a coactivator of AP-1}

Based on the genetic interactions described above, we proceeded to analyze the epistatic relationship between Chm and AP-1 factors. To this end, we used UAS transgenes and the MZ980Gal4 driver that promotes expression specifically in the proximal region of the wing disc and later along the prospective junction of the contralateral discs (Martin-Blanco et al. 2000). Driving UASchm in these cells fully rescues the thoracic cleft of $\mathrm{chm} \mathrm{mu-}$ tants, while Dfos, Diun, or both Dfos and Diun expression in the MZ980 domain do not (Fig. 3A). Thus, chm is not epistatic to $D$ fos or Diun, which indicates that $\mathrm{Chm}$ is not upstream of AP-1 in the pathway and does not control the expression of Dfos and Diun. RT-PCR from RNA samples of wild-type and chm mutant wing discs confirmed that Dfos and Diun transcription is not affected by $\mathrm{chm}$ loss of function (data not shown). This finding is consistent with Chm cooperating with JNK signaling at the level of the transcriptional effectors of the pathway.

Then, we investigated whether Chm directly binds to DFos and/or DJun using GST pull-down assays. The Cterminal half of Chm (amino acids 494-812), which contains the MYST domain, displays strong in vitro affinity for an $\mathrm{N}$-terminal fragment of DFos (including the $\mathrm{N}$ terminus and the basic DNA-binding domain), and binds DJun as well, although less efficiently. Conversely, the cytoplasmic kinase Basket (Bsk)/DJNK does not bind to the His-Chm fusion protein (Fig. 3B). The Chm N terminus (amino acids 20-400) does not associate with DFos or DJun (data not shown). Similar experiments with GST-fused DFos deletion mutants identified the basic region of DFos as the predominant Chm-interacting do- 
Miotto et al.

Figure 3. Chm acts as a nuclear coactivator of DJun and DFos. (A) Rescue of chm thoracic cleft phenotype by Chm, DFos, DJun, or DJun plus DFos expression in the MZ980 domain. Refer to Figure 1A for the color code of phenotypic classes. (B) GST pull-down assays. Binding of His-Chm ${ }^{\text {Cter }}$ to GST fusions of DJun, DFos $^{\mathrm{N} \text {-ter }}$ or DJNK was revealed by Western blot probed by anti-His and anti-GST antibodies, and secondary antibodies coupled to peroxydase (brown) and alkaline phosphatase (blue). (C) Immunoprecipitation of nuclear extracts from chm; 69BGal4/UASMyc-chm third instar larvae using IgG or anti-Myc. Immunoprecipitates were washed by increasing salt concentration and analyzed by Western blot using antibodies to DFos, DJun, Mod, En, Ubx, and DMyc as a negative control. $(D)$ Western blots probed with anti-Myc of immunoprecipitates obtained by anti-TAP from nuclear extracts of larvae ubiquitously expressing MycChm and TAP-DFos or Myc-Chm and TAPDJun, or of wild-type larvae (Mock). (E) Luciferase assays of HEK293 cells transfected with the reporter construct and combinations of expression vectors for DJNKK, DJNK, DFos, DJun, and Chm (50, 100, and $250 \mathrm{ng} /$ well). Histograms represent luciferase activity relative to a value of 1 for transfection with reporter alone (error bars show standard deviations, five independent assays). (F) Luciferase assays with DJNKK, DJNK, DFos, DJun, and hTip60 (250 $\mathrm{ng} /$ well). Reference value of 1 in the absence of hTip60; four independent assays. (Inset) Western blot probed with anti-Flag of cell extracts transfected with a Flag-hTip60-producing or empty vector.
A
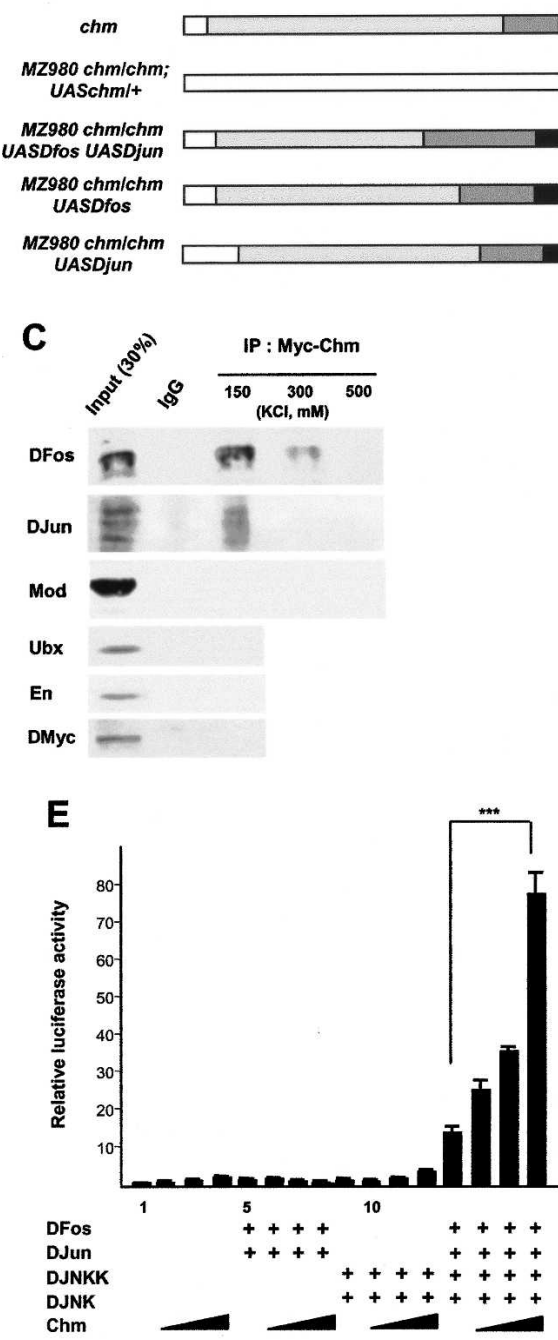

B

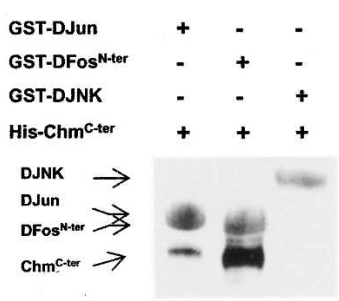

D
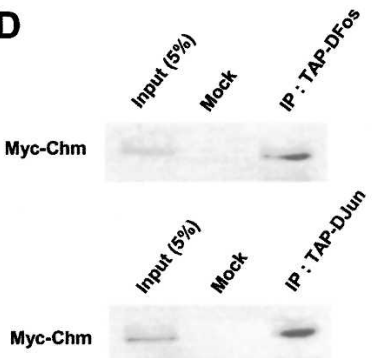

$\mathbf{F}$

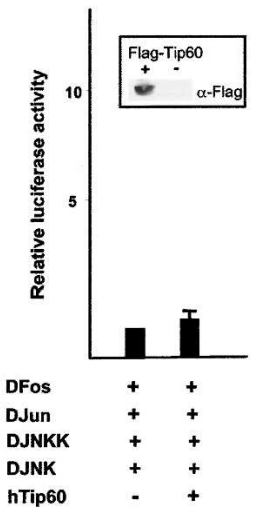

main, although significant association with the C-terminal part of DFos was also observed (see Supplementary Fig. S1). Immunoprecipation assays followed by Western analyses confirmed that these interactions occur in vivo. Both DFos and DJun coprecipitate with Myc-Chm from nuclear extracts of larvae expressing a Myc-tagged version of the protein (Fig. 3C). DFos is eluted from the immunoprecipitate at higher salt concentrations than DJun, which indicates a more stable association with Chm and that Chm to DFos interaction can occur in the absence of DJun. Confirming the specificity of the assay, nuclear proteins unrelated to JNK signaling, the chromatin-associated protein Modulo (Mod), the homeodomain transcription factors Ultrabithorax (Ubx) and Engrailed (En), as well as the basic helix-loop-helix (bHLH) factor DMyc are not precipitated by Myc-Chm (Fig. 3C). In reciprocal experiments, an anti-TAP antibody coprecipitates Myc-Chm from nuclear extracts of larvae ubiquitously expressing Myc-Chm and TAP-DFos or TAP-DJun (Fig. 3D). The results of the in vitro and the in vivo experiments, taken together, show that Chm, DFos and DJun can directly interact and form multimeric protein complexes in larvae.
To further characterize the cooperation between DFos, DJun and Chm, we performed reporter gene assays in cultured cells. HEK293 cells were transiently transfected with an AP-1-dependent luciferase reporter in the presence or absence of Chm, DJun and DFos, DJNKK and DJNK. Chm either alone, or in the presence of DFos and DJun or of DJNKK and DJNK, had no significant effect (Fig. 3E). However, when DJNKK, DJNK, DFos, and DJun were supplied together, Chm stimulated AP-1-dependent transcription in a dose-dependent manner. Thus, Chm promotes the transactivation potential of AP-1 only when the JNK pathway is active. This effect seems to be specific for Chm, as expression of hTip60, another MYST HAT (Hilfiker et al. 1997), does not change luciferase activity (Fig. 3F). These data identify Chm as a transcriptional coactivator of DFos and DJun.

\section{DFos recruits Chm to chromatin to promote transcriptional activity}

Next, we focused on the functional relevance of the $\mathrm{Chm} / \mathrm{DF}$ os interaction. To investigate whether this in- 
teraction occurs on AP-1 target DNA, we performed chromatin immunoprecipitation (ChIP) assays in HEK293 cells transfected with an AP-1 reporter and constructs for HA-DFos and Flag-Chm. DFos efficiently and specifically binds the reporter promoter. Chm does so only when expressed together with DFos (Fig. 4A). No significant binding was observed on the vector DNA. To ascertain that Chm and DFos simultaneously associated with promoter sequences, we performed a sequential ChIP experiment. After a first ChIP with an antibody to HA (DFos) and dissociation of the antibodies from the immunoprecipitated complexes, a second ChIP was performed using anti-Flag (Chm) antibody. PCR detection then showed that the complex eventually pulled-down contains both DFos and Chm associated with the promoter (Fig. 4A, bottom). As a control, Chm but not hTip60 was coprecipitated by DFos from cells transfected with HA-DFos and either Flag-Chm or FlaghTip60 (Fig. 4B). We conclude that $\mathrm{Chm}$ is recruited to chromatin in a sequence specific manner by DFos to AP1-binding sites.

Next, we asked whether Chm acts by promoting DFos DNA-binding or transactivating potential. This was addressed in an in vivo assay, using a fly line that expresses a chimera between the DNA-binding LexA protein and DFos, and permits monitoring transactivation by DFos independent of its DNA-binding activity (Szuts and Bienz 2000). We observed that a lacZ reporter cloned downstream of LexA-binding sites is strongly activated in the larval epidermis by DFos-LexA and that chm mutation reduces lacZ transcription (Fig. 4C). This result suggests that $\mathrm{Chm}$ supports gene activation in the animal by a mechanism improving DFos transactivation capacity.

\section{INK-mediated phosphorylation of DFos regulates the functional interaction with $\mathrm{Chm}$}

So far, we have seen that DFos and Chm physically interact and functionally cooperate in the execution of JNK signals to mediate apoptosis, morphogenesis, and gene activation. Next, we addressed how JNK influences the function of DFos and Chm at the molecular level. DFos is a substrate for JNK and several phosphorylation sites have been reported (Ciapponi et al. 2001). The relevance of JNK-mediated phosphorylation of DFos in thorax closure is illustrated by the strong cleft phenotype induced by the expression in the MZ980 domain of a
A

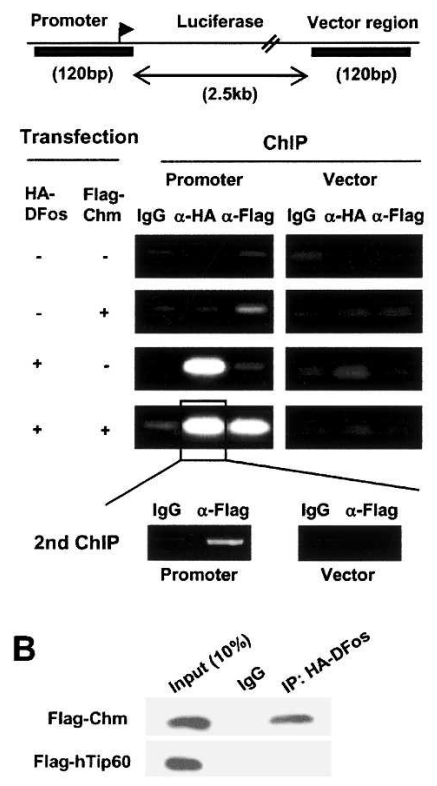

C

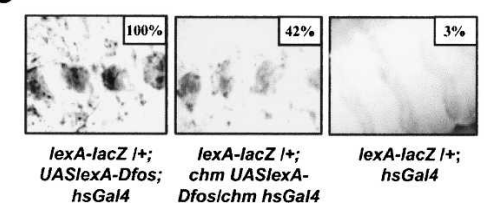

D

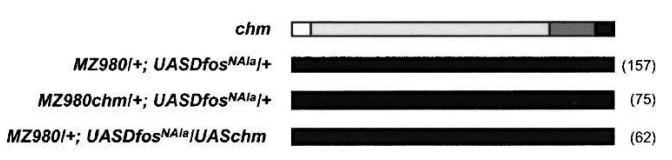

E

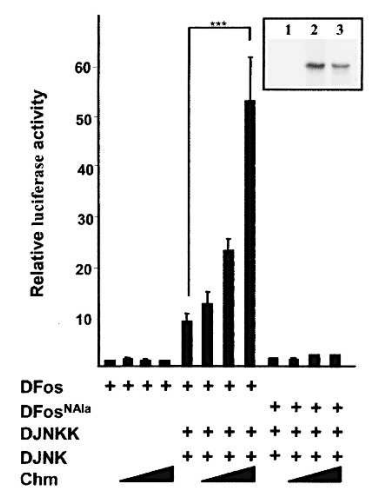

$\mathbf{F}$

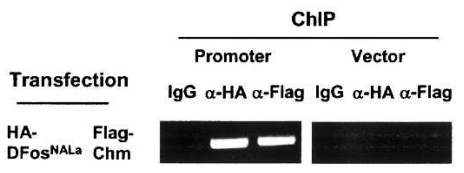

Figure 4. Cooperation between $\mathrm{Chm}$ and DFos in transcriptional activation. $(A)$ ChIP with IgG, anti-HA, and anti-Flag of chromatin extracts from HEK293 cells transfected with vectors producing FlagChm, HA-DFos, or both. (Top) Occupancy was assayed by real-time PCR using primers for regions (shown in black) encompassing AP-1-binding sites (left) or a vector region (right). (Bottom) Second ChIP with anti-Flag from chromatin complexes precipitated by anti-HA. (B) Western blots probed with anti-Flag of immunoprecipitates obtained by anti-HA or IgG from nuclear extracts of HEK293 cells transfected with vectors producing HA-DFos and Flag-Chm (top) or HA-DFos and FlaghTip60 (bottom). (C) Magnified views of larval epidermis stained for $\beta$-galactosidase upon lexA-lacZ reporter induction by LexA-DFos chimera in an otherwise wildtype (left) or chm mutant (middle) background, and of lexA-lacZ animals as a control (right). Levels of $\beta$-galactosidase expression are given as the percentage of the value obtained for lexA-lacZ/+; UASLexADfos; hsGal4 larvae. (D) Lack of genetic interactions of $\mathrm{chm}$ with the $D$ fos $^{\text {NAla }}$ variant during thorax closure. Refer to Figure $1 \mathrm{~A}$ for the color code of phenotypic classes. (E) Luciferase assays of HEK293 cells transfected with DFos and Chm (50, 100 , and $250 \mathrm{ng} /$ well) in the presence or absence of JNK and DJNKK, and with DFos ${ }^{\text {NAla }}$ and Chm in the presence of JNK and DJNKK. Reference value of 1 for transfection with reporter alone, four independent assays. (Inset) Western blot, probed with anti-DFos antibody, of cell extracts transfected with an empty vector (lane 1) and with vectors producing DFos ${ }^{\text {NAla }}$ (lane 2) or DFos (lane 3). (F) ChIP with IgG, anti-HA, and anti-Flag of chromatin extracts from HEK293 cells transfected with vectors producing Flag-Chm and HA-DFos ${ }^{\text {NAla }}$. Occupancy was assayed by real-time PCR using primers for promoter and vector regions. 
variant, DFos ${ }^{\mathrm{NAla}}$, in which the two DJNK threonine residues that represent DJNK targets were mutated into alanine (Fig. 4D). Interestingly, this effect cannot be reversed by $\mathrm{Chm}$ overexpression, indicating that the protein cannot promote gene activation by DFos ${ }^{\mathrm{NAla}}$. To further validate this interpretation, we performed reporter gene assays in HEK293 cells. The luciferase reporter is activated by DFos, DJNKK and DJNK, and in this context Chm expression boosts transcription in a concentration-dependent manner whereas no such effect is observed using DFos ${ }^{\text {NAla }}$ instead of the wild-type protein (Fig. 4E). These data strongly suggest that the cooperation between DFos and Chm in gene activation is regulated by a mechanism that involves DFos phosphorylation. Interestingly, phosphorylation by DJNK does not seem to be required for Chm to DFos binding as DFos $^{\text {Nala }}$ recruits Chm to chromatin as efficiently as the wild-type protein (Fig. 4F).

\section{Chm promotes $H 4$ acetylation at AP-1 target sites}

The recruitment of Chm to AP-1-binding sites by DFos suggests a mechanism for gene activation that involves histone modification by the HAT activity of Chm. As the bZIP domain of DFos is sufficient to recruit Chm, and to avoid the additional effects of the transactivation and phosphorylation domains of DFos, we used a truncated version of DFos that essentially comprises the bZIP domain. Thus, we devised a simple experimental system relying on DFos ${ }^{\text {bZIP }}$ expression in HEK293 cells to explore the mode of action of Chm.

We first tested the requirement of Chm HAT activity for DFos-mediated transcription. Coexpression of DFos $^{\text {bZIP }}$ with Chm results in a significant stimulation of reporter activity. This is dependent on the enzymatic activity of $\mathrm{Chm}$, because a point mutation in the acetylCoA-binding site (G680E) that abolishes HAT activity (Grienenberger et al. 2002) abrogates reporter activation (Fig. 5A). The effect of $\mathrm{Chm}^{\mathrm{G} 680 \mathrm{E}}$ is dominant as indicated by luciferase inhibition when the level of the mutant protein is raised leaving that of the wild-type constant. In these experiments, similar levels of mutant and wild-type Chm proteins are expressed (data not shown) and bind chromatin in transfected HEK293 cells (Fig. 5B). The dominant negative effect of $\mathrm{Chm}^{\mathrm{G} 680 \mathrm{E}}$ was also observed using the full-length DFos protein /data not shown).

To assess whether HAT-dependent AP-1 reporter activation correlated with changes in histone modification, we next examined the acetylation pattern at the reporter gene promoter and its dependence on $\mathrm{Chm}$ enzymatic activity by performing ChIP experiments with acetylation site-specific antibodies. Chm recruitment by DFos ${ }^{\text {bZIP }}$ results in an increase of $\mathrm{H} 4$ tetra-acetylation but does not change K9/K14 acetylation of H3, while Chm ${ }^{\text {G680E }}$ has no significant effect (Fig. 5C). Thus, H4 is a likely target of DFos-directed acetylation by Chm. In ChIP assays with antibodies to mono-acetylated $\mathrm{H} 4$ isoforms, Chm was found to enhance the H4K16 acetylation over levels observed in the presence of DFos ${ }^{\text {bZIP }}$
A

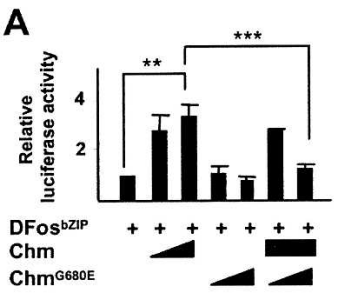

B
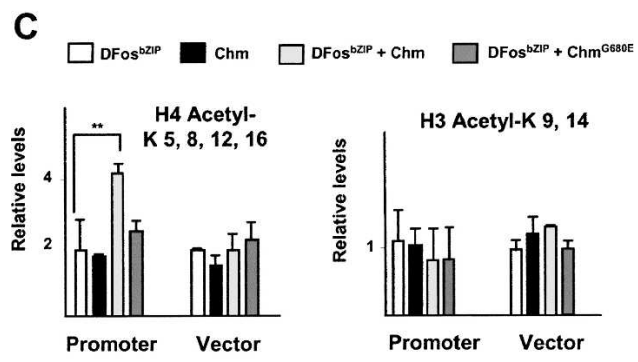

D
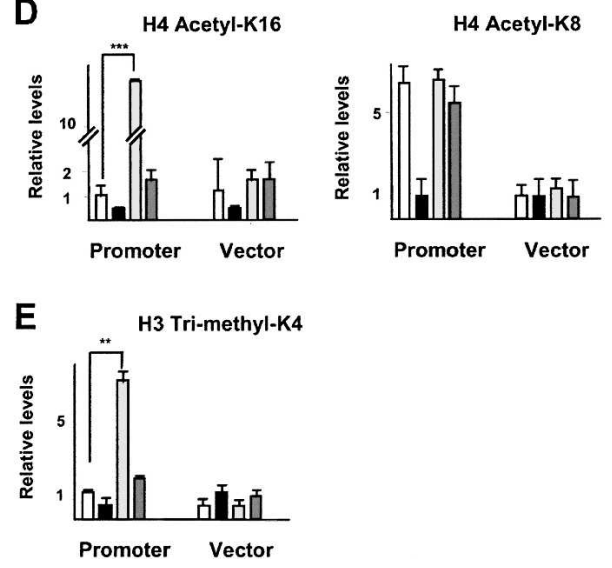

Figure 5. Chm promotes $\mathrm{H} 4$ acetylation at AP-1-responsive sequence. (A) Luciferase assays of HEK293 cells transfected with DFos ${ }^{\text {bZIP }}, \mathrm{Chm}$, and/or $\mathrm{Chm}^{\mathrm{G} 680 \mathrm{E}}$ (100 or $250 \mathrm{ng} /$ well). Reference value of 1 for transfection with DFos ${ }^{\text {bZIP }}$ alone; three independent assays. $(B)$ Western blots, probed with antibody to Flag and HA tags, of chromatin preparations from cells transfected with vectors producing HA-DFos ${ }^{\text {bZIP }}$ and Flag-Chm or Flag-Chm ${ }^{\mathrm{G} 680 \mathrm{E}}$. (C-E) ChIP of chromatin extracts from HEK293 cells transfected with DFos ${ }^{\text {bZIP }}$, Chm, DFos ${ }^{\text {bZIP }}$ and Chm, or DFos $^{\text {bZIP }}$ and $\mathrm{Chm}^{\mathrm{G} 680 \mathrm{E}}$. Immunoprecipitations were performed by antibodies to tetra-acetylated $\mathrm{H} 4(C, l e f t)$, acetylated $\mathrm{H} 3 \mathrm{~K} 9 /$ $\mathrm{K} 14(C$, right $)$, acetylated $\mathrm{H} 4 \mathrm{~K} 16(D$, left), acetylated H4K8 (D, right), and trimethylated H3K4 $(E)$. Data of real-time PCR are given as relative levels compared with cells transfected with reporter alone (average of four independent assays).

alone, an effect that is not supported by Chm ${ }^{\mathrm{G} 680 \mathrm{E}}$ (Fig. 5D). Thus, H4K16 is a target lysine of Chm HAT activity. Interestingly, DFos ${ }^{\text {bZIP }}$ promotes H4K8 acetylation but Chm addition does not raise the level further. This indicates that $\mathrm{H} 4 \mathrm{~K} 8$ is not a Chm target, and therefore suggests that DFos ${ }^{\text {bZIP }}$ recruits another HAT to modify this lysine.

Then, we examined Chm function in transcription activation. H3K4 trimethylation constitutes a chromatin mark for transcriptionally active loci (Lachner and Jenuwein 2002). Consistent with the coactivator function of 
Chm, the level of H3K4 trimethylation at the promoter is increased in the presence of both Chm and DFos ${ }^{\mathrm{bZIP}}$. Again, Chm HAT activity is required in this process, as using the Chm ${ }^{\mathrm{G} 680 \mathrm{E}}$ variant has no effect (Fig. 5E). This suggests that $\mathrm{H} 4$ acetylation by $\mathrm{Chm}$ is a prerequisite for the definition of a histone modification pattern instructive for improved transcription.

\section{DRpd3 interacts with DFos and counteracts Chm function}

The identification of HAT activities as mediators of DFos-dependent histone acetylation and gene activation raises the question of which HDACs might function to counter these processes. A hypomorphic mutation of the gene encoding the Drosophila homolog of HDAC1, Drpd3, dominantly suppresses the thoracic cleft of chm mutants, whereas available mutations in other HDAC genes, including DHDAC4 and Dsir2, have no effect (Fig. 6A). Furthermore, heterozygosity for Drpd3 enhances the hep ${ }^{C A}$ notched wing phenotype and counteracts the phenotypic rescue caused by the mutation of one copy of chm (Fig. 6B). Thus, Drpd3 and chm antagonistically control JNK signaling during thorax closure and the JNKdependent apoptotic pathway. As H4K8 is modified by another HAT than Chm (Fig. 5D), we also tested genetic interactions with available mutations in HAT genes. Reducing the gene dosage of nejire/CBP, mof, enok, or deco neither aggravated nor rescued the chm thorax phenotype (Fig. 6A).

To examine the effect of DRpd3 on AP-1-dependent transcription at the molecular level, we asked whether DRpd3 could directly interact with DFos, as Chm does. DRpd3 coprecipitates with TAP-DFos from nuclear ex- tracts of larvae expressing UASTAP-Dfos under the control of armGal4 (Fig. 6C). GST pull-down assays reveal that DFos physically interacts with DRpd3 and that the only DFos fragments that precipitate DRpd3 share the ZIP domain (Supplementary Fig. S1). Thus, DRpd3 and Chm physically interact with the ZIP and the basic domains of DFos, respectively. Finally, to assess the functional relevance of these interactions, we tested the effect of DRpd3 expression on DFos ${ }^{\text {bZIP }} / \mathrm{Chm}$-induced transcription and histone modifications in HEK293 cells. Increasing DRpd3 levels while leaving Chm constant results in reporter repression (Fig. 6D). Consistent with this inhibitory effect, DRpd3 reduces $\mathrm{H} 4$ tetra-acetylation and H3K4 trimethylation mediated by DFos ${ }^{\text {bZIP } / ~}$ Chm at the promoter (Fig. 6E), indicating that DRpd3 restores a repressive transcriptional environment. Thus, Chm and DRpd3 behave as antagonistic cofactors of DFos $^{\text {bZIP }}$ in HEK293 cells. Considered together, these data strongly suggest that the nuclear response to JNK signaling is determined by the opposing activities of this HAT/HDAC pair.

\section{Regulation of Chm and DRpd3 recruitment and function by INK signaling}

To investigate the roles of Chm and DRpd3 in JNK phosphorylation-dependent transactivation, we performed experiments with the full-length version of DFos. To modulate JNK activity we employed sorbitol treatment, which can reversibly activate the pathway in cultured cells (Bagowski et al. 2003). In HEK293 cells transfected with Chm, DRpd3 and DFos vectors, luciferase transcription rapidly increases after sorbitol addition and is progressively shut-down after sorbitol removal (Fig. 7A).
A

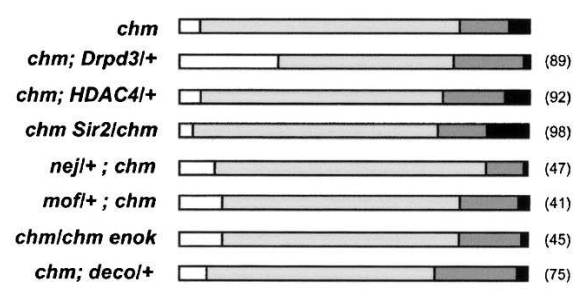

C

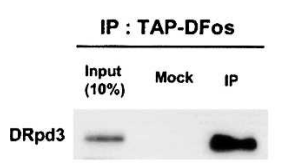

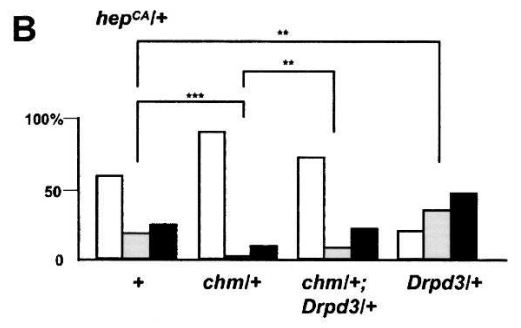

$(100)$

(154) (84)

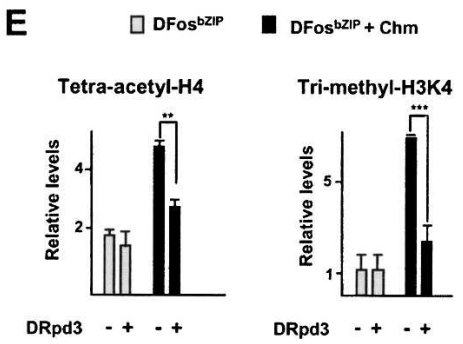

Figure 6. DRpd3 interacts with DFos and counteracts $\mathrm{Chm}$ function. (A) Genetic interactions of chm with HDAC- and HATencoding genes during thorax closure, revealed by the strength of the thoracic cleft phenotype. The numbers of examined pupae are given in parentheses. Refer to Figure $1 \mathrm{~A}$ for the color code of phenotypic classes. (B) Changes in the distribution of the wing phenotype classes associated with hep ${ }^{C A}$ (see Fig. 2A) by heterozygosis for $\operatorname{Drpd} 3^{04556}$ allele and suppression of the rescuing effect of $\mathrm{chm}$ heterozygosis. Refer to Figure 2A for the color code of phenotypic classes. $(C)$ Western blots, revealed by anti-DRpd3, of immunoprecipitates obtained by anti-TAP from nuclear extracts of wild-type (Mock) and armGal4 UASTAP-Dfos/UASMyc-chm third instar larvae. $(D)$ Luciferase assays of HEK293 cells transfected with DFos ${ }^{\text {bZIP }}$, Chm $(250$ ng/well), and increasing amounts of DRpd3 (50, 100, or $250 \mathrm{ng} /$ well). Reference value of 1 for cells transfected with DFos ${ }^{\text {bzIP }}$ alone; four independent assays. (E) ChIP of chromatin extracts from HEK293 cells transfected with DFos ${ }^{\text {bZIP }}$ or DFos ${ }^{\text {bZIP }}$ and Chm, in the presence or absence of DRpd3. Immunoprecipitations were performed with antibodies to tetra-acetylated H4 (left) and to trimethylated H3K4 (right). Data are represented as a relative levels compared with transfection with the reporter alone (average of three independent assays). 
Miotto et al.

A
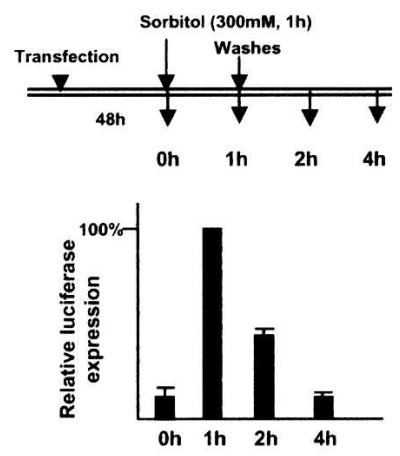

NT Oh 1h 2h $4 \mathrm{~h}$

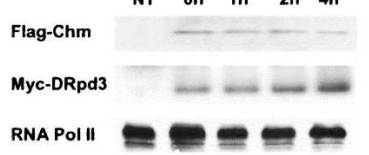

D

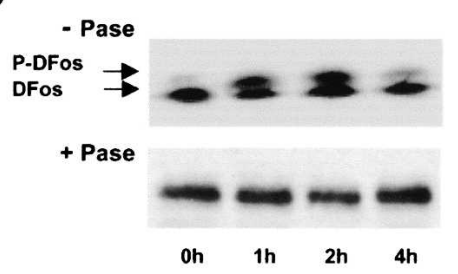

B

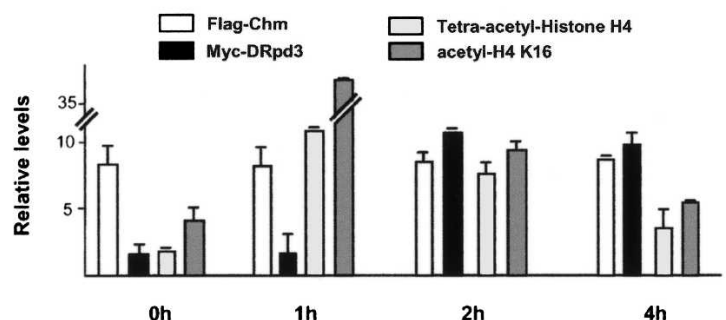

C
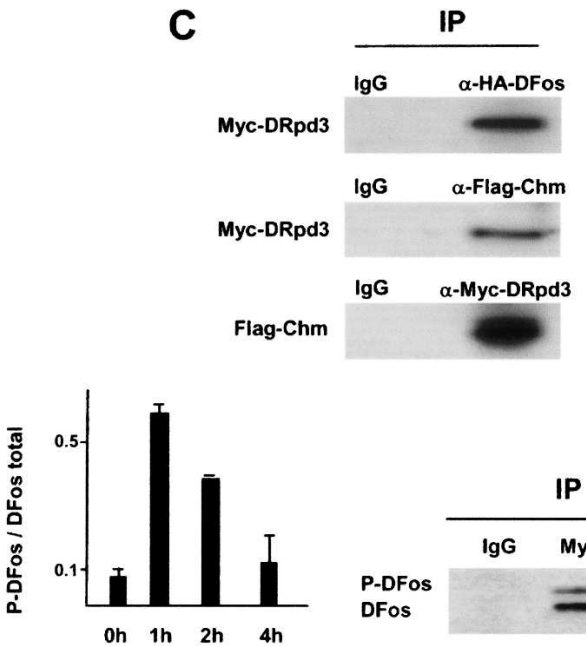

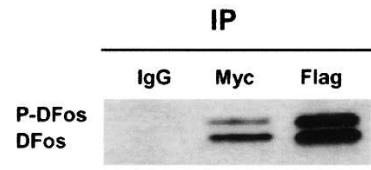

E

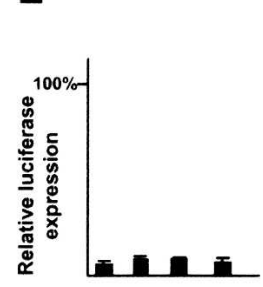

oh 1 h $2 \mathrm{~h} \quad 4 \mathrm{~h}$

F
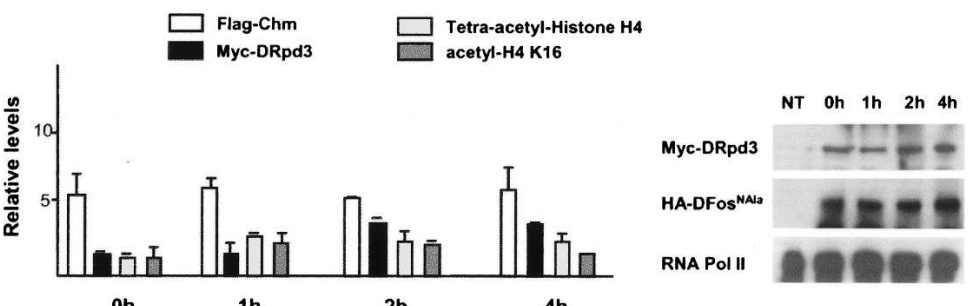

Figure 7. Chm and DRpd3 recruitment and function upon JNK signaling activation and deactivation. $(A-C)$ Compilation of data obtained from RT-PCR, ChIP, and Western blot analyses during JNK signaling activation and inhibition upon sorbitol addition $(300$ $\mathrm{mM}$ for $1 \mathrm{~h}$ ) and removal in HEK293 cells transfected with HA-DFos, Flag-Chm, and Myc-DRpd3. (A, top) At each time point described, total RNA was harvested for RT-PCR to access luciferase transcription, and chromatin extract was prepared for ChIP analyses. (Middle) luciferase transcription. (Bottom) Control Western blots of cell extracts for Chm and DRpd3 expression, and RNA pol II as a loading control. (NT) Nontransfected cells. (B) ChIP with anti-Flag, anti-Myc, anti-tetra-acetylated H4, and anti-acetyled H4K16 antibodies. RT-PCR data are normalized relative to GAPDH expression. ChIP data are depicted as relative levels compared with transfection with the reporter alone (average of three independent assays). (C) Western blots, probed for proteins indicated to the left, of proteins associated with chromatin fragments extracted $1 \mathrm{~h}$ after sorbitol removal and immunoprecipitated by antibodies to Flag $(\mathrm{Chm})$, Myc (DRpd3), or HA (DFos). (D, left) Phosphorylation status of chromatin-bound HA-DFos was assessed by Western blot of chromatin samples probed by anti-HA. +Pase and -Pase refer to chromatin samples digested or not with calf intestinal alkaline phosphatase. (Middle) Time course upon sorbitol addition and removal of P-DFos/total DFos ratio obtained by phosphoimager gel scan. (Right) Western blot for DFos and P-DFos of chromatin fragments extracted $1 \mathrm{~h}$ after sorbitol removal and immunoprecipitated by Myc-DRpd3 and Flag-Chm. $(E, F)$ Compilation of data obtained from similar experiments as in $A$ and $B$, but using HA-DFos ${ }^{\text {NAla }}$ instead of HA-DFos (average of four independent assays). (Right) Western blots of cell extracts for DRpd3, DFos ${ }^{\text {NAla }}$, and RNA pol II.

Therefore, reversible stimulation by sorbitol constitutes an appropriate system to correlate molecular changes at AP-1-responsive promoter with JNK-dependent transcription.

We performed ChIP assays to assess changes in reporter promoter occupancy throughout the experiment
(Fig. 7B). We found that $\mathrm{Chm}$ is always and at invariant levels associated with the promoter, indicating that its recruitment by DFos does not depend on JNK signaling. $\mathrm{H} 4$ acetylation levels at the promoter, as monitored by the relative abundance of $\mathrm{H} 4 \mathrm{~K} 16$ and tetra-acetylated $\mathrm{H} 4$, dynamically changed throughout the time course of 
induction and correlated with transcriptional activity (Fig. 7B). Because DFos-directed acetylation by $\mathrm{Chm}$ is responsible for these modifications (Fig. 5D), this result indicates that the protein, while present at the promoter, is unable to acetylate $\mathrm{H} 4$ in nonstimulated cells, and therefore that JNK signaling stimulates the latent HAT activity of $\mathrm{Chm}$ and permits subsequent transcriptional activation.

Whereas Chm is found constitutively associated, DRpd3 recruitment to the promoter depends on JNK signaling. Before sorbitol addition, which we could consider as the inactive steady state, DRpd3 does not associate the promoter (1.35-fold over background). DRpd3 thus appears dispensable for maintenance of the inactive steady state. While activating the pathway upon sorbitol addition has no significant effect on DRpd3 tethering, the HDAC starts to be recruited to the promoter after sorbitol elimination (14-fold over background $1 \mathrm{~h}$ after sorbitol removal) and the levels of $\mathrm{H} 4$ acetylation and transcription then decrease simultaneously and progressively. Thus, the down-regulation of the transcriptional response to JNK activation after dissipation of the signal is at least in part mediated by recruitment of DRpd3 to the promoter already occupied by $\mathrm{Chm}$. Supporting this notion, DRpd3 is found on chromatin fragments immunoprecipitated by $\mathrm{Chm}$ and conversely $\mathrm{Chm}$ is associated with fragments containing DRpd3 (Fig. 7C). Together, these results suggest that DRpd3 assumes a transient function to reduce histone modification and transcription levels previously stimulated by Chm.

The dynamics of Chm activation and DRpd 3 recruitment to the promoter was next considered with regard with DFos phosphorylation. Sorbitol addition increases the ratio of phosphorylated DFos (P-DFos) to nonphosphorylated DFos in the chromatin-bound fraction, and sorbitol removal decreases the ratio (Fig. 7D). Thus, DFos phosphorylation correlates with Chm-mediated transcriptional activation and DFos dephosphorylation with down-regulation by DRpd3. Furthermore, Western analysis of immunoprecipitated chromatin revealed that $1 \mathrm{~h}$ after sorbitol removal Chm, DFos and P-DFos are present on the same chromatin fragments, whereas DRpd3 coprecipitates predominantly with fragments containing unphosphorylated DFos (Fig. 7D, right). Pulldown assays indicate that DFos interacts in vitro with both Chm and DRpd3, and P-DFos only with Chm (see Supplementary Fig. S2). Together, these data suggest that the DFos phosphorylation state is the likely connection between sorbitol stimulus, Chm HAT activity unmasking and DRpd3 recruitment. To investigate this point further, we performed sorbitol experiments using the DFos $^{\text {NAla }}$ variant, which cannot be phosphorylated, instead of the wild-type protein. Consistent with our earlier finding (Fig. 4E), Chm still is tethered to the promoter. However, sorbitol addition no longer stimulates $\mathrm{H} 4$ acetylation and target gene transcription (Fig. 7E,F). This lack of JNK responsiveness correlates with a significantly reduced change in promoter occupancy by DRpd3 in response to JNK signaling. Thus, the DFos phosphorylation status controls Chm HAT activity and DRpd3 recruitment to the promoter.

\section{Discussion}

\section{Chm/DRpd3 partnership in the developmental} response to JNK signaling

This study establishes a direct link between JNK signaling and chromatin modification via AP-1 transcription factors in an intact developing organism, an effect that rests on the HAT coactivator $\mathrm{Chm}$ and the HDAC corepressor DRpd3. This conclusion holds for thoracic closure and JNK-induced apoptosis but not for another JNKdependent morphogenetic event, the embryonic dorsal closure. Although this process shares many similarities with thorax closure (Kockel et al. 2001), it is not affected in embryos deprived of maternal and zygotic chm contribution (data not shown). Thus, $\mathrm{Chm}$ is not required for all JNK signaling-dependent mechanisms. Furthermore, when it is involved, Chm is not essential for JNK signaling: The incomplete penetrance of $\mathrm{chm}$ phenotypes and the decreased but remaining expression of AP-1 target genes in mutant discs illustrate that chm loss-offunction compromises but does not block JNK signaling. Thus, Chm is not an obligatory component in the pathway but its recruitment supports the transcriptional efficiency of AP-1 during imaginal development. DRpd3 counteracts this function, indicating that the balanced activity of the two antagonistic factors constitutes a new regulatory mechanism for JNK-dependent transcription.

The functional partnership between Chm and DRpd3 in the control of JNK signaling seems specific to this HAT/HDAC pair, as mutations in other HDAC or HAT genes do not modify the chm thorax closure phenotype. Particularly illustrative is the absence of genetic interactions with mutant alleles of mof, since Mof is a MYST HAT of the same substrate specificity as Chm-H4K16 (Akhtar and Becker 2000)—and DFos interacts with Chm through the conserved MYST domain. Interestingly, a recent report has connected $\mathrm{Chm}$ and DRpd3 functions during Drosophila oogenesis (Aggarwal and Calvi 2004). The two proteins exhibit opposite effects on $\mathrm{H} 4$ acetylation and activity of replication origins, indicating that the functional antagonism of $\mathrm{Chm}$ and $\mathrm{DRpd} 3$ regulates several chromatin-dependent processes, including transcription and replication.

Although genetic experiments emphasize the specificity of Chm/DRpd3 partnership, our data do not imply that DRpd3's only role in JNK signaling is in balancing $\mathrm{Chm}$ function. Neither do they exclude that other HDACs nor HATs participate in the JNK response. For example, in cultured cells DRpd3 recruitment by AP-1 changes histone acetylation levels established earlier by the HAT Pcaf at the attacin promoter from a permissive to a repressive status (Kim et al. 2005), and a HDAC3 repressor complex maintains c-Jun transcriptionally inactive (Weiss et al. 2003). It is therefore likely that different HATs and HDACs control AP-1-regulated tran- 
scription, depending on the physiological context. What specifies HAT and HDAC commitment in a given process remains to be explored.

\section{Mechanisms of Chm/DRpd3-mediated control of AP-1-dependent transcription}

Insights into the mechanism by which Chm supports JNK target gene transcription were initially obtained from observations made in the developing animal. First, Chm improves the activation of a lexA-lac $Z$ reporter by the LexA-DFos fusion protein, revealing that $\mathrm{Chm}$ stimulates the transactivating potential of DFos when it is tethered to a promoter. Second, the thorax cleft phenotype caused by DFos ${ }^{\mathrm{NAla}}$, a protein deficient for the JNK phosophorylation sites, is not rescued upon simultaneous overexpression of $\mathrm{Chm}$. As the DFos ${ }^{\mathrm{NAla}}$ variant recruits Chm as efficiently as the wild-type protein, this suggests that transcriptional improvement by Chm requires DFos phosphorylation, providing an attractive link between Chm function and JNK signaling.

Our study of the mode of action of the antagonistic cofactors Chm and DRpd3 in cultured cells has provided further insights into a chromatin-based mechanism that executes a modulation of the transcriptional response to JNK signaling. We propose the following model, based on the dynamics of cofactor recruitment and activity, chromatin modification and transcriptional status related to reversible activation of the pathway. In response to JNK signaling, Chm HAT activity sets up a histone modification pattern that is instructive for transcriptional enhancement. Consistent with this notion Chm acetylates $\mathrm{H} 4$, with a marked preference for K16, and facilitates H3K4 trimethylation. However, AP-1 likely also engages HATs of different substrate specificity, since H4K8 acetylation, a modification required for the recruitment of the SWI/SNF-activating complex (Agalioti et al. 2002), is directed by DFos in the absence of Chm. After JNK signaling has ceased, DRpd3 gets recruited to promoters occupied by unphosphorylated DFos and counteracts the effects of Chm by reversing histone modifications, which results in transcriptional down-regulation. Strikingly, the recruitment of DRpd3 seems not to result from the displacement of Chm from the promoter, since invariant levels of $\mathrm{Chm}$ are associated with the promoter in sorbitol experiments, whereas DRpd3 starts to be recruited only once the signal has been eliminated. Thus, as opposed to an exchange of a HAT coactivator complex for an HDAC corepressor complex, which occurs for instance between Pcaf/NF-kb and DRpd3/AP-1 complexes at the attacin promoter (Kim et al. 2005), a complex containing both $\mathrm{Chm}$ and $\mathrm{DRpd} 3$ could then form at the target promoter whose activity changes the histone modification pattern back to a pattern less permissive to transcription. Thus, DRpd3 most likely functions during a transient phase from a transcriptionally active to silent status. Its absence from the promoter at the inactive steady state in nonstimulated cells, suggests that unphosphorylated DFos then lies in a conformational envi- ronment that prevents DRpd3 recruitment by the ZIP domain.

AP-1 phosphorylation plays a pivotal role in regulating the epigenetic response to JNK signaling. An "activation by derepression" model has been recently proposed following which JNK-dependent phosphorylation induces the release of an HDAC3 repressor complex from the $\mathrm{N}$ terminus of c-Jun (Weiss et al. 2003; Ogawa et al. 2004). We have seen that DFos ${ }^{\text {bZIP }}$, which lacks JNK phosphorylation sites and domains other than the bZIP that could recruit HDAC complexes, can promote transcription in nonstimulated cells. This suggests that a phosphorylation-dependent process similar to the activation by derepression model mediates transactivation by fulllength DFos when JNK signaling is active. Our work furthermore supports additional regulatory roles for AP-1 phosphorylation as indicated by the dependence of Chm HAT activity and DRpd3 recruitment on the DFos phosphorylation state. We propose that phosphorylation not only releases an HDAC corepressor complex (other than DRpd3) and leads to activation by derepression but also unmasks Chm HAT function and results in increased transcriptional efficiency, whereas dephosphorylation promotes the recruitment of DRpd3 to reverse transcriptional activation. Considering the opposite functions of chm and Drpd3 in JNK-dependent thorax closure and apoptosis during fly development, it is tempting to speculate that the balanced activities of the Chm HAT and the DRpd3 HDAC allow transient JNK target gene activation as well as fine-tuning of JNK transcriptional output in vivo.

\section{Materials and methods}

Fly stocks and manipulations

The following fly lines were used: UASLexA-Dfos and LL lexAlacZ reporter construct (M. Bienz, Cambridge, UK); $\mathrm{kay}^{1}, \mathrm{kay}^{2}$, Diun $^{\text {IA109 }}$, UASDiun, UASDfos, UASDfos ${ }^{\text {NAla }}$, UASTAP-Djun, and UASTAP-Dfos (D. Bohmann); deco ${ }^{1}$ (M.L. Goldberg, Ithaca, $\mathrm{NY}$ ); $\operatorname{mof}^{1}$ (J.C. Lucchesi, Atlanta, GA); $\operatorname{enok}^{1}$ (L. Luo, Stanford, CA); MZ980Gal4 (E. Martin-Blanco, Barcelona, Spain); hep ${ }^{\text {A }}$ and $\mathrm{Omb}^{b i}$ (K. Matsumoto, Nagoya, Japan); hep ${ }^{1}$ (S. Noselli, Nice, France); DSir2 ${ }^{05327}$ (S.M. Parkhurst, Nice, France); the null $\mathrm{chm}^{14}$ allele (Grienenberger et al. 2002) and $\mathrm{chm}^{\text {7lacz }}$ (J. Pradel); DHDAC4 ${ }^{\text {KG05226 }}$,Drpd3 ${ }^{04556}$, nej $^{3}$, puc $^{E 69}$, sd $^{\text {Gal4 }}$, armGal4, 69BGal4, and hsGal4 (Bloomington). UASMyc-chm was generated using a Myc-tag/chm cDNA fusion cloned in pUAS-T, and UASMyc-chm ${ }^{\text {G680E }}$ by replacing an Age1/Xbal fragment of pUAS-T-Mycchm by the homologous fragment with G680E point mutation. In situ hybridizations and immunodetections were performed according to standard procedures. Digoxigenin RNA-labeled probes were generated according to manufacturer's protocol (Boehringer-Mannheim). All primer sequences used in this work are available on request.

Larval nuclear extract preparation, immunoprecipitation, and $\beta$-galactosidase activity quantification

Nuclei were isolated from third instar larvae using a slightly modified version of the protocol of Lehmann and Koolman (1988). Nuclear preparations (10 mM Tris; 0.1 mM EDTA; 150 $\mathrm{mM} \mathrm{KCl} ; 10 \%$ glycerol; $1 \mathrm{mM} \mathrm{DTT}$, leupeptin, aprotinin, and pepstatin protease inhibitors at $\mathrm{pH} 7.5$ ) were immunoprecipi- 
tated by anti-Myc or rabbit-IgG coupled to agarose beads (Sigma) for $2 \mathrm{~h}$ at $4^{\circ} \mathrm{C}$. Beads were washed five times with increasing salt concentrations (150-500 mM KCl), boiled in Laemmli buffer and eluted proteins subjected to $10 \%$ SDS-PAGE, transferred onto nitrocellulose membrane, and probed with monoclonal antibodies to the Myc Tag (Santa Cruz), Mod (Garzino et al. 1992), En (4D9, Developmental Studies Hybridoma Bank), Ubx (FP3.38), and DMyc (Prober and Edgar 2000) or polyclonal antibodies to DFos (Zeitlinger and Bohmann 1999), DJun (Santa Cruz), or HDAC1/DRpd3 (Abcam). Quantitative assays of $\beta$-galactosidase activity were performed as described in Simon and Lis (1987), and the values obtained from triplicate assays on batches of 20 larvae of each genotype are given as a relative percentage, setting to $100 \%$ the value obtained for $1 e x A-l a c Z /+$; UASLexA-Dfos; hsGal4 animals.

\section{Cell culture transfection and luciferase assays}

HEK293 cells were grown in DMEM with 10\% FBS and transiently transfected using the calcium phosphate precipitation method. Expression vectors pAP-1-Luciferase, pCEFL-HADFos, pCEFL-HA-DJun, pCEFL-HA-DJNK, and pCEFL-HADJNKK were kindly provided by S.X. Hou (Frederick, MD), and pCMV2-hTip60 was provided by K.V. Kandror (Boston, MA). Vectors for Flag-Chm and Flag-Chm ${ }^{\mathrm{G} 680 \mathrm{E}}$ expression were generated from PCR-amplified $\mathrm{chm}$ and $\mathrm{chm}^{\text {G680E }}$ cDNAs cloned in frame in pXJ-Flag. pDEST26-HA-Fos ${ }^{\text {bZIP }}$, pDEST26-HA-Fos ${ }^{\text {NAla }}$, and pDEST26-Myc-DRpd3 expression vectors were obtained by PCR amplification of the corresponding coding sequences. All constructs were sequence-verified. Reporter plasmid $(1 \mu \mathrm{g})$ and expression vectors (50 ng, except when stated otherwise) were transfected with $100 \mathrm{ng}$ of CMV- $\beta$-Gal plasmid, and total DNA was scaled up to $10 \mu \mathrm{g}$ with pBluescript. For luciferase assays, cells were lysed in $300 \mu \mathrm{L}$ of lysis buffer $(25 \mathrm{mM}$ Tris, $2 \mathrm{mM}$ EDTA, 1 mM DTT, 10\% Glycerol, $1 \%$ Triton X-100 at pH 7.8) by three cycles of freeze-thawing, and extracts were analyzed for $\beta$-Gal $(30 \mu \mathrm{L})$ or luciferase $(50 \mu \mathrm{L})$ activities. For immunoprecipitations, nuclear extracts from transfected HEK293 cells were treated as described above.

\section{GST pull-down assays}

GST fusions DFos ${ }^{\mathrm{A}}$, DFos ${ }^{\mathrm{D}}$, DJun, and DJNK are described in Ciapponi et al. (2001). His-Chm ${ }^{\text {Nter }}$ and His-Chm ${ }^{\text {Cter }}$ were generated by subcloning BamH1/StuI (Nter) or StuI/HindIII (Cter) fragments of chm cDNAs in pQE31. DFos ${ }^{\mathrm{B}}, \mathrm{DFos}^{\mathrm{C}}$, and DFos ${ }^{\mathrm{E}}$ deletions were generated by PCR amplification and Gateway recombination. Recombinant proteins were expressed in Escherichia coli BL21. ${ }^{35}$ S-methionine-labeled DRpd3 was produced by in vitro transcription/translation according to manufacturer's instructions (Promega). Pull-down assays were adapted from Ciapponi et al. (2001), using $300 \mathrm{mM} \mathrm{NaCl}$ and $1.2 \mu \mathrm{g} / \mathrm{mL}$ of ethidium bromide in the binding buffer.

\section{ChIP analyses}

Assays were performed with soluble chromatin from $2 \times 10^{8}$ HEK293 cells. Antibodies to modified histones were from Upstate Biotechnology and from Abcam. Anti-HA-agarose, antiMyc-agarose, IgG-agarose, and anti-Flag-Sepharose were from Sigma. ChIP assays were performed as in Cawley et al. (2004). To ascertain that variations in histone modification levels were not due to nucleosome displacement, removal, or enrichment, controls by ChIP with anti-H4 were systematically performed. No significant changes in nucleosome occupancy were observed.

\section{RT-PCR assays}

RNA from wing imaginal discs (1 h APF) or transfected HEK293 cells was extracted by Trizol (GIBCO-BRL) and purified by RNeasy column (Qiagen). One microgram of total RNA was used for first-strand cDNA reaction using oligo $\mathrm{d}(\mathrm{T})$ and SuperScript II reverse transcriptase (Invitrogen) for $50 \mathrm{~min}$ at $42^{\circ} \mathrm{C}$. RNA quantification by real-time PCR was performed as described above, and normalized with reference to $r p 49$ RNA for wing imaginal discs and GAPDH for HEK293 cells.

\section{Statistical analyses}

Statistical analyses were performed with the Student's $t$-test: ns indicates no statistical difference, $P<0.6 ;{ }^{*}, P<0.1$; **, $P<0.01$; and ${ }^{\star \star \star}, P<0.001$.

\section{Acknowledgments}

We thank A.J. Saurin for critical reading of the manuscript, P. Ramain for initial help in cell culture, and colleagues and the Bloomington centre for fly lines. This work was supported by the CNRS, grants from "l'Association pour la Recherche contre le Cancer" (ARC) and "la Ligue Nationale Contre Le Cancer" (LNCC), and fellowships from "le Ministère de la Recherche et de la Technologie" (MRT) and l'ARC.

\section{References}

Adachi-Yamada, T., Fujimura-Kamada, K., Nishida, Y., and Matsumoto, K. 1999. Distortion of proximodistal information causes JNK-dependent apoptosis in Drosophila wing. Nature 400: 166-169.

Agalioti, T., Chen, G., and Thanos, D. 2002. Deciphering the transcriptional histone acetylation code for a human gene. Cell 111: 381-392.

Aggarwal, B.D. and Calvi, B.R. 2004. Chromatin regulates origin activity in Drosophila follicle cells. Nature 430: 372-376.

Agnes, F., Suzanne, M., and Noselli, S. 1999. The Drosophila JNK pathway controls the morphogenesis of imaginal discs during metamorphosis. Development 126: 5453-5462.

Akhtar, A. and Becker, P.B. 2000. Activation of transcription through histone $\mathrm{H} 4$ acetylation by MOF, an acetyltransferase essential for dosage compensation in Drosophila. Mol. Cell 5: 367-375.

Bagowski, C.P., Besser, J., Frey, C.R., and Ferrell Jr., J.E. 2003. The JNK cascade as a biochemical switch in mammalian cells: Ultrasensitive and all-or-none responses. Curr. Biol. 13: 315-320.

Bannister, A.J. and Kouzarides, T. 1995. CBP-induced stimulation of c-Fos activity is abrogated by E1A. EMBO J. 14: 47584762 .

Benkoussa, M., Brand, C., Delmotte, M.H., Formstecher, P., and Lefebvre, P. 2002. Retinoic acid receptors inhibit AP-1 activation by regulating extracellular signal-regulated kinase and CBP recruitment to an AP-1-responsive promoter. Mol. Cell Biol. 22: 4522-4534.

Cawley, S., Bekiranov, S., Ng, H.H., Kapranov, P., Sekinger, E.A., Kampa, D., Piccolboni, A., Sementchenko, V., Cheng, J., Williams, A.J., et al. 2004. Unbiased mapping of transcription factor binding sites along human chromosomes 21 and 22 points to widespread regulation of noncoding RNAs. Cell 116: 499-509. 
Ciapponi, L., Jackson, D.B., Mlodzik, M., and Bohmann, D. 2001. Drosophila Fos mediates ERK and JNK signals via distinct phosphorylation sites. Genes \& Dev. 15: 1540-1553.

Eferl, R. and Wagner, E.F. 2003. AP-1: A double-edged sword in tumorigenesis. Nat. Rev. Cancer 3: 859-868.

Garzino, V., Pereira, A., Laurenti, P., Graba, Y., Levis, R.W., Le Parco, Y., and Pradel, J. 1992. Cell lineage-specific expression of modulo, a dose-dependent modifier of variegation in Drosophila. EMBO J. 11: 4471-4479.

Grienenberger, A., Miotto, B., Sagnier, T., Cavalli, G., Schramke, V., Geli, V., Mariol, M.C., Berenger, H., Graba, Y., and Pradel, J. 2002. The MYST domain acetyltransferase Chameau functions in epigenetic mechanisms of transcriptional repression. Curr. Biol. 12: 762-766.

Hilfiker, A., Hilfiker-Kleiner, D., Pannuti, A., and Lucchesi, J.C. 1997. mof, a putative acetyltransferase gene related to the Tip60 and MOZ human genes and to the SAS genes of yeast, is required for dosage compensation in Drosophila. EMBO J. 16: 2054-2060.

Jasper, H., Benes, V., Schwager, C., Sauer, S., Clauder-Munster, S., Ansorge, W., and Bohmann, D. 2001. The genomic response of the Drosophila embryo to JNK signaling. Dev. Cell 1: 579-586.

Kim, T., Yoon, Y., Cho, H., Lee, W-B., Kim, J., Song, Y-H., Kim, S.N., Yoon, J.H., Kim-Ha, J., and Kim, Y-J. 2005. Downregulation of lipopolysaccharide response in Drosophila by negative crosstalk between the AP-1 and the NF-B signaling modules. Nat. Immunol. 6: 211-218.

Kockel, L., Homsy, J.G., and Bohmann, D. 2001. Drosophila AP-1: Lessons from an invertebrate. Oncogene 20: 23472364.

Lachner, M. and Jenuwein, T. 2002. The many faces of histone lysine methylation. Curr. Opin. Cell Biol. 14: 286-298.

Lehmann, M. and Koolman, J. 1988. Ecdysteroid receptors of the blowfly Calliphora vicina: Partial purification and characterization of ecdysteroid binding. Mol. Cell Endocrinol. 57: 239-249.

Liu, X., Grammont, M., and Irvine, K.D. 2000. Roles for scalloped and vestigial in regulating cell affinity and interaction between the wing blade and wing hinge. Dev. Biol. 228: 287303.

Martin-Blanco, E., Pastor-Pareja, J.C., and Garcia-Bellido, A. 2000. JNK and decapentaplegic signaling control adhesiveness and cytoskeleton dynamics during thorax closure in Drosophila. Proc. Natl. Acad. Sci. 97: 7888-7893.

Munz, C., Psichari, E., Mandilis, D., Lavigne, A.C., Spiliotaki, M., Oehler, T., Davidson, I., Tora, L., Angel, P., and Pintzas, A. 2003. TAF7 (TAFII55) plays a role in the transcription activation by c-Jun. J. Biol. Chem. 278: 21510-21516.

Noselli, S. and Agnes, F. 1999. Roles of the JNK signaling pathway in Drosophila morphogenesis. Curr. Opin. Genet. Dev. 9: 466-472.

Ogawa, S., Lozach, J., Jepsen, K., Sawka-Verhelle, D., Perissi, V., Sasik, R., Rose, D.W., Johnson, R.S., Rosenfeld, M.G., and Glass, C.K. 2004. A nuclear receptor corepressor transcriptional checkpoint controlling activator protein 1-dependent gene networks required for macrophage activation. Proc. Natl. Acad. Sci. 101: 14461-14466.

Pastor-Pareja, J.C., Grawe, F., Martin-Blanco, E., and GarciaBellido, A. 2004. Invasive cell behavior during Drosophila imaginal disc eversion is mediated by the JNK signaling cascade. Dev. Cell 7: 387-399.

Prober, D.A. and Edgar, B.A. 2000. Ras1 promotes cellular growth in the Drosophila wing. Cell 100: 435-446.

Simon, J.A. and Lis, J.T. 1987. A germline transformation analysis reveals flexibility in the organization of heat shock con- sensus elements. Nucleic Acid Res. 15: 2971-2988.

Szuts, D. and Bienz, M. 2000. LexA chimeras reveal the function of Drosophila Fos as a context-dependent transcriptional activator. Proc. Nat1. Acad. Sci. 97: 5351-5356.

Weiss, C., Schneider, S., Wagner, E.F., Zhang, X., Seto, E., and Bohmann, D. 2003. JNK phosphorylation relieves HDAC3dependent suppression of the transcriptional activity of cJun. EMBO T. 22: 3686-3695.

Weston, C.R. and Davis, R.J. 2002. The JNK signal transduction pathway. Curr. Opin. Genet. Dev. 12: 14-21.

Xia, Y. and Karin, M. 2004. The control of cell motility and epithelial morphogenesis by Jun kinases. Trends Cell. Biol. 14: 94-101.

Zeitlinger, J. and Bohmann, D. 1999. Thorax closure in Drosophila: Involvement of Fos and the JNK pathway. Development 126: 3947-3956.

Zhang, J., Kalkum, M., Chait, B.T., and Roeder, R.G. 2002. The N-CoR-HDAC3 nuclear receptor corepressor complex inhibits the JNK pathway through the integral subunit GPS2. Mol. Cell 9: 611-623. 


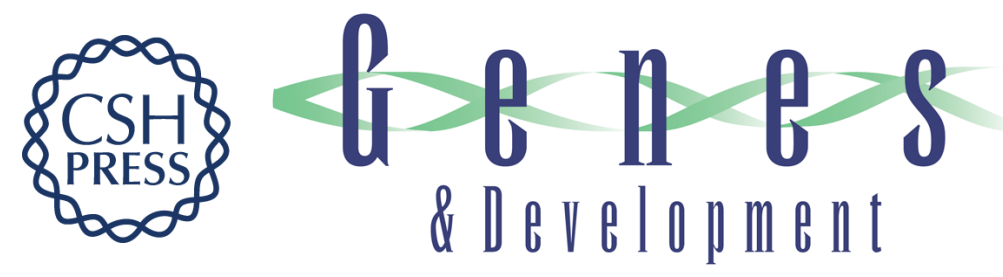

\section{Chameau HAT and DRpd3 HDAC function as antagonistic cofactors of JNK/AP-1-dependent transcription during Drosophila metamorphosis}

Benoit Miotto, Thierry Sagnier, Hélène Berenger, et al.

Genes Dev. 2006, 20:

Access the most recent version at doi:10.1101/gad.359506

Supplemental
Material $\quad$ http://genesdev.cshlp.org/content/suppl/2005/12/30/20.1.101.DC1

References This article cites 33 articles, 10 of which can be accessed free at:

http://genesdev.cshlp.org/content/20/1/101.full.html\#ref-list-1

License

Email Alerting Receive free email alerts when new articles cite this article - sign up in the box at the top

Service right corner of the article or click here.

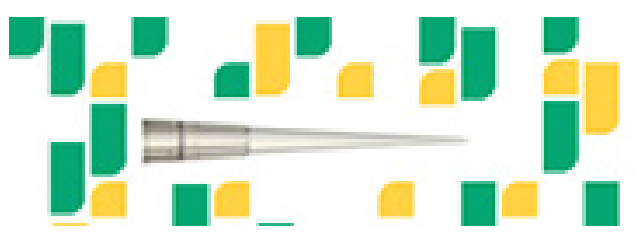

Focused on your science. 